\title{
An Automatic Sensing Setup for Methamphetamine Based on the Reactional Wettability Variation
}

\section{Strategy}

Yang Zhao, ${ }^{[a] *}$ Yahang Xie, ${ }^{[b]}$ Qifan Zhou, ${ }^{[b]}$ Pan Wang, ${ }^{[b]}$ Ying Chang, ${ }^{[a]}$ Changxu Lin ${ }^{[b] *}$

[a] Institute of Forensic Science, Ministry of Public Security, 100038, Beijing, China

[b] Research Institute for Biomimetics and Soft Matter, Fujian Provincial Key Laboratory for Soft Functional Materials Research, College of Physical Science and Technology, Xiamen University, 9 Zengcuoan West Road, 361005 Xiamen, China

Table S1. Calibration of recognized contact angle value vs. measure from contact angle meter.

\begin{tabular}{lll}
\hline Example Droplet & CA recognized & CA from contact angle meter \\
\hline 1 & $60.5^{\circ} \pm 1.2^{\circ}$ & $58.6^{\circ} \pm 0.2^{\circ}$ \\
2 & $102.7^{\circ} \pm 2.4^{\circ}$ & $100.5^{\circ} \pm 1.4^{\circ}$ \\
3 & $118.6^{\circ} \pm 3.4^{\circ}$ & $116.4^{\circ} \pm 2.7^{\circ}$ \\
4 & $122.3^{\circ} \pm 3.8^{\circ}$ & $119.5^{\circ} \pm 3.4^{\circ}$ \\
\hline
\end{tabular}

The Source Code of robotic movement and droplet control \# encoding: utf-8 
(a)Author: XIe

@Email: 565831006@qq.com

@File: droplet.py

@Time: 2020/10/09 17:02

"'"'"

from HitbotInterface import HitbotInterface

import time

import serial

import threading

import struct

\#move funtion definition

class pump_serial:

def _ init_ (self, port, baudrate $=9600$, timeout $=1$ ):

self.port $=$ serial. Serial $($ port, baudrate $)$

if self.port.is_open:

print("pump'port is open")

else:

print("pump'port is close")

def i_move(self, volume, i_rate):

writeintxt $=$ 'cvolume $\backslash r$ '

self.port.write(writeintxt.encode())

writeintxt $=$ 'tvolume ' $+\operatorname{str}($ volume $)+{ }^{\prime} \mathrm{ml}+$ ' 'r'

self.port.write(writeintxt.encode()) 


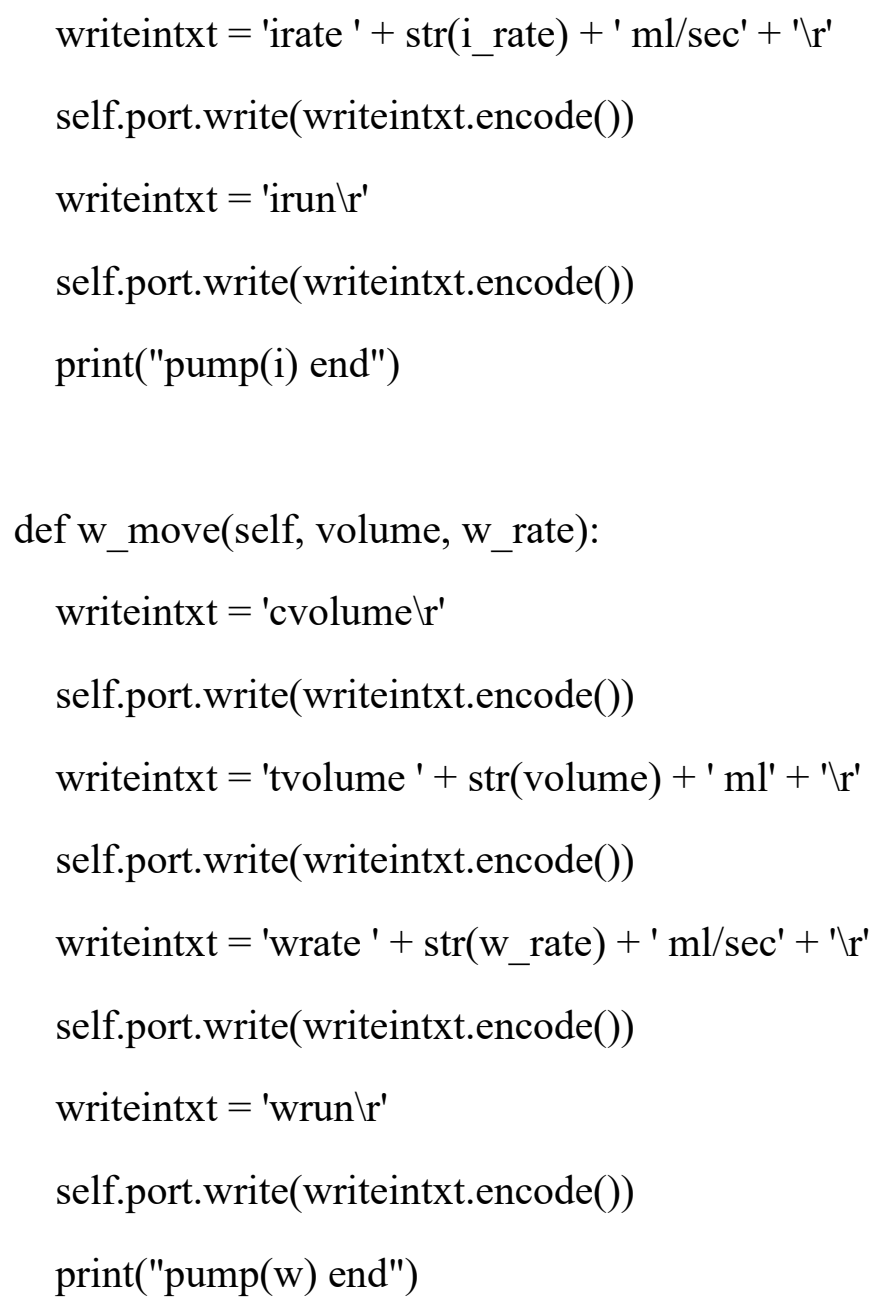




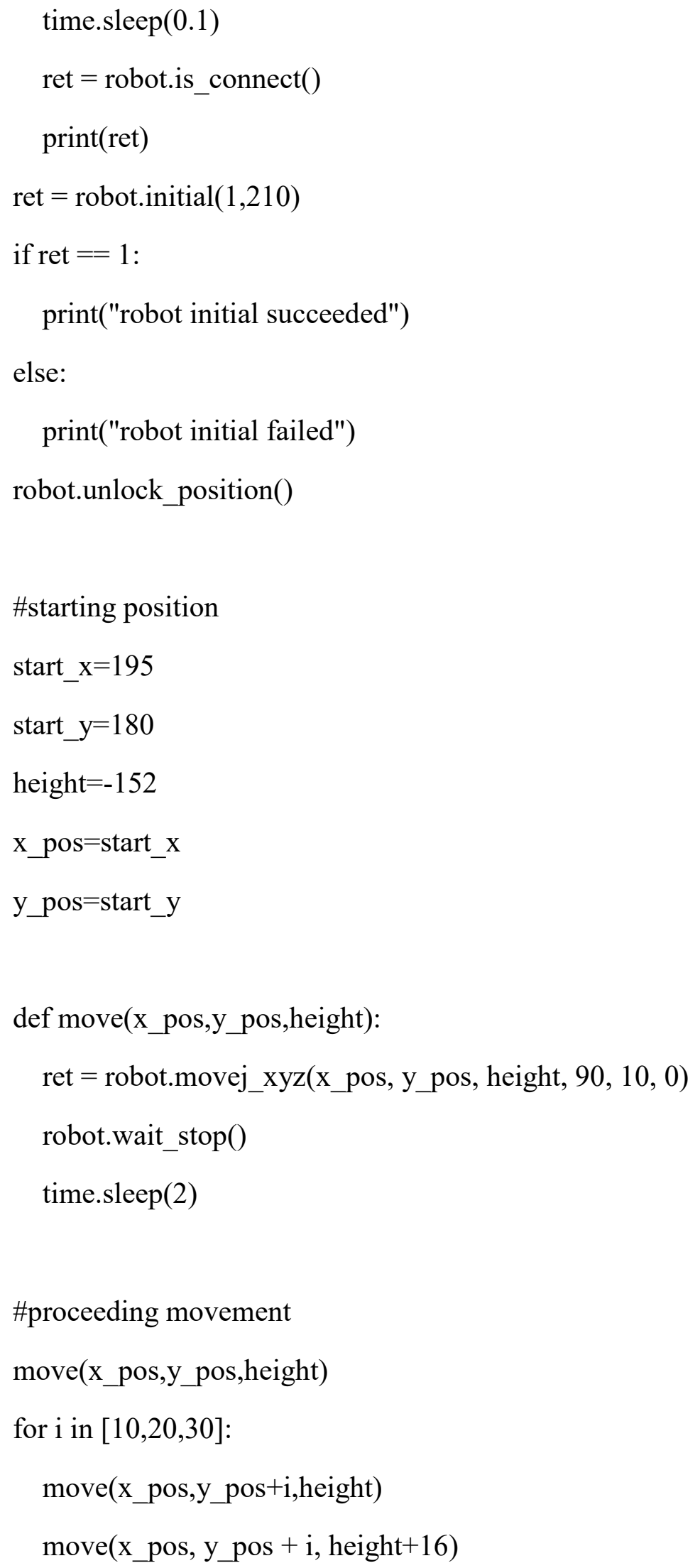


pump.i_move $(0.0004,0.0002)$

time.sleep (5)

move(x_pos, y_pos $+\mathrm{i}$, height +12$)$

move(x_pos, y_pos $+\mathrm{i}$, height)

move(x_pos,y_pos,height)

print("end") 